\title{
Deriving physical parameters of unresolved star clusters ${ }^{\star}$
}

\section{M 31 PHAT star clusters}

\author{
P. de Meulenaer ${ }^{1,2}$, R. Stonkute $\dot{e}^{1,2}$, and V. Vansevičius ${ }^{1,2}$ \\ ${ }^{1}$ Center for Physical Sciences and Technology, Saulètekio av. 3, 10257 Vilnius, Lithuania \\ 2 Vilnius University Observatory, Čiurlionio 29, 03100 Vilnius, Lithuania \\ e-mail: vladas.vansevicius@ff.vu.lt
}

Received 8 March 2017 / Accepted 29 March 2017

\begin{abstract}
Context. This study is the fifth of a series that investigates the degeneracy and stochasticity problems present in the determination of physical parameters such as age, mass, extinction, and metallicity of partially resolved or unresolved star cluster populations in external galaxies when using HST broad-band photometry.

Aims. In this work we aim to derive parameters of star clusters using models with fixed and free metallicity based on the HST WFC3+ACS photometric system. The method is applied to derive parameters of a subsample of 1363 star clusters in the Andromeda galaxy observed with the HST.

Methods. Following Paper III, we derive the star cluster parameters using a large grid of stochastic models that are compared to the six observed integrated broad-band WFC3+ACS magnitudes of star clusters.

Results. We show that the age, mass, and extinction of the M 31 star clusters, derived assuming fixed solar metallicity, are in agreement with previous studies. We also demonstrate the ability of the WFC3+ACS photometric system to derive metallicity of star clusters older than $\sim 1$ Gyr. We show that the metallicity derived using broad-band photometry of 36 massive M 31 star clusters is in good agreement with the metallicity derived using spectroscopy.
\end{abstract}

Key words. galaxies: individual: M31 - galaxies: star clusters: general

\section{Introduction}

In this study we derive star cluster parameters by applying stochastic models to real star clusters from the Andromeda galaxy. Using the Wide Field Camera 3 (WFC3) and the Advanced Camera for Surveys (ACS) on board the Hubble Space Telescope (HST), the Panchromatic Hubble Andromeda Treasury (PHAT) team (see, e.g. Dalcanton et al. 2012, Beerman et al. 2012, Weisz et al. 2013) performed a photometric survey of one third of the M31 galaxy. Based on the PHAT survey a large "year 1" catalogue of 601 star clusters was provided by Johnson et al. (2012). Fouesneau et al. (2014) derived the age, mass, and extinction of the clusters from this catalogue using the aperture photometry data (Johnson et al. 2012). They used a constant solar metallicity throughout the whole M 31 disk, arguing that the HII zone study (Zurita \& Bresolin 2012) does not show any significant metallicity gradient. However, they allowed four different metallicities $([\mathrm{M} / \mathrm{H}]=-0.7,-0.4,0.0$, and +0.4 , that is, from Small Magellanic Cloud to super-solar metallicities) for 30 massive globular-like clusters with a mass $>10^{5} M_{\odot}$.

In de Meulenaer et al. (2015a) we reanalyzed this cluster sample by first fixing the metallicity of all clusters to the solar value, and a second time by allowing a large range of metallicities in the model grid with 13 values of $[\mathrm{M} / \mathrm{H}]=-2.2$ to +0.2 , in steps of 0.2 . When using fixed metallicity, we found a satisfactory agreement for the majority of clusters between

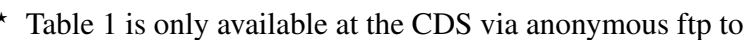
cdsarc.u-strasbg. fr (130.79.128.5) or via http://cdsarc.u-strasbg.fr/viz-bin/qcat?J/A+A/602/A112
}

the parameters derived in our study and those supplied by Fouesneau et al. (2014). When leaving the metallicity free, we found in many cases older ages, especially for globular-like clusters. However, for clusters with a high enough signal-tonoise ratio we derived metallicities in agreement with the spectroscopically derived ones by Caldwell et al. (2011). Therefore, our analysis confirmed a high potential of the WFC3+ACS photometric system to study semi-resolved and unresolved star clusters.

Recently the PHAT star cluster survey was completed by Johnson et al. (2015) and published in a final catalogue containing 2753 clusters. The objectives followed here are: to select a subsample of clusters from the Johnson et al. (2015) catalogue with a high enough photometric accuracy to derive cluster age, mass, extinction, and metallicity, and to compare the derived parameters with the spectroscopic studies by Caldwell et al. (2009, 2011), Caldwell \& Romanowsky (2016).

The structure of the paper is the following: Sect. 2 presents the method of star cluster parameter determination using the WFC3+ACS photometric system. Section 3 presents the application of the method on the PHAT star clusters. In this section, the derived cluster parameters are compared to the values derived in the spectroscopic studies. Section 4 discusses some issues of cluster parameter determination based on aperture photometry. Conclusions are presented in Sect. 5.

\section{Method of cluster parameter determination}

Following Fouesneau \& Lançon (2010), Fouesneau et al. (2014), and de Meulenaer et al. (2013; 2014; 2015a; 2015b; hereafter 

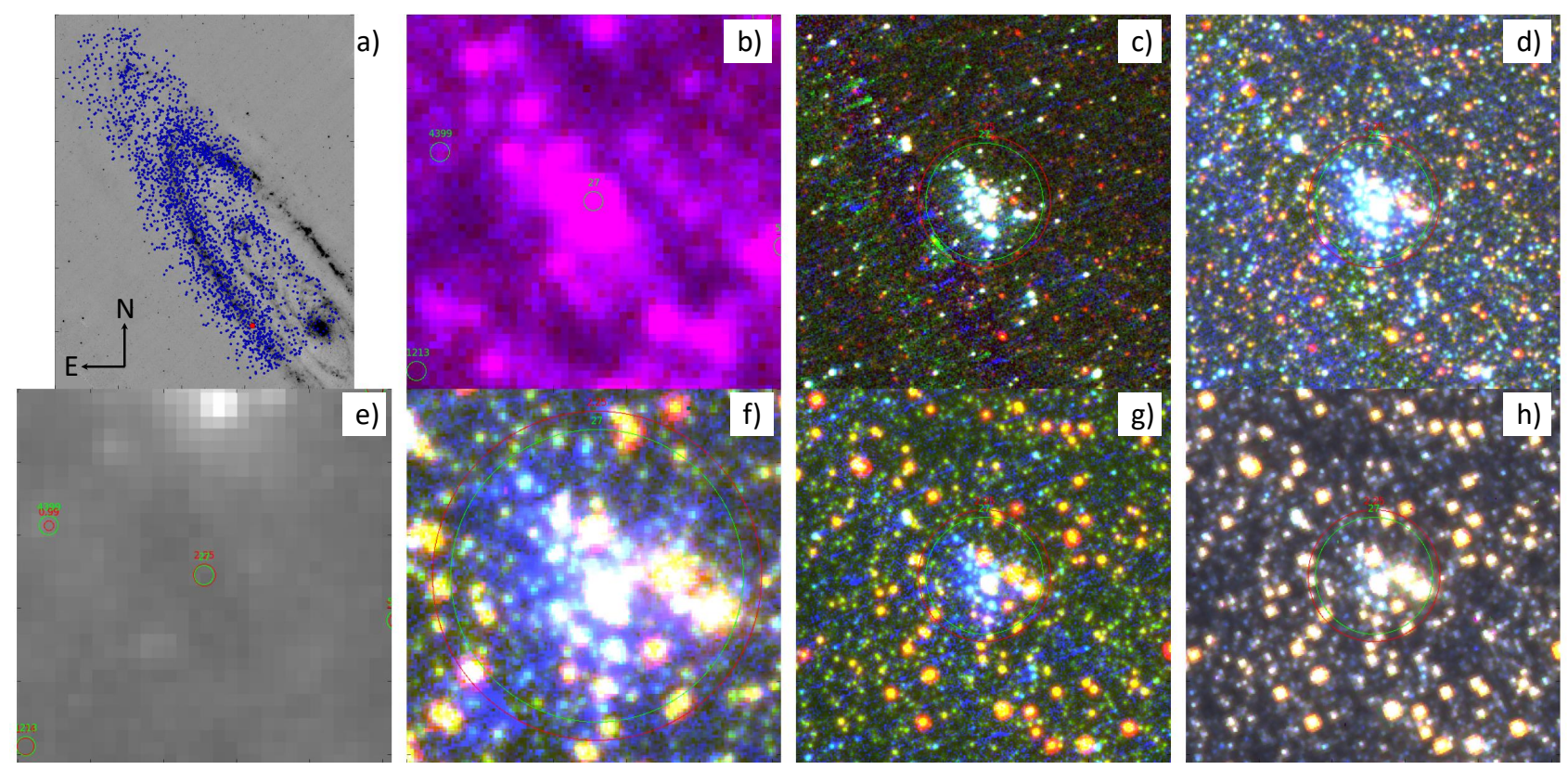

Fig. 1. Sky images constructed in different photometric passbands for the cluster ID27. Multiband images in the WFC3+ACS passbands are made from the PHAT mosaics downloaded at the MAST (Mikulski Archives for Space Telescopes) ${ }^{1}$. Panels show combinations of passbands: a) PHAT clusters (blue dots) plotted on the Spitzer $24 \mu \mathrm{m}$ map - the cluster under consideration is marked with a red dot; b) GALEX FUV (blue) + NUV (red) $\left(76^{\prime \prime} \times 76^{\prime \prime}\right)$; c) $F 275 W+F 336 W+F 475 W\left(13^{\prime \prime} \times 13^{\prime \prime}\right)$; d) $F 336 W+F 475 W+F 814 W\left(13^{\prime \prime} \times 13^{\prime \prime}\right)$; e) the $24 \mu$ m passband centred on the cluster $\left(76^{\prime \prime} \times 76^{\prime \prime}\right)$; f) $F 336 W+F 814 W+F 110 W\left(5^{\prime \prime} \times 5^{\prime \prime}\right)$; g) $\left.F 275 W+F 814 W+F 160 W\left(13^{\prime \prime} \times 13^{\prime \prime}\right) ; \mathbf{h}\right) F 814 W+F 110 W+F 160 W$ $\left(13^{\prime \prime} \times 13^{\prime \prime}\right)$. In panels $\left.\left.\left.\left.\mathbf{c}\right), \mathbf{d}\right), \mathbf{f}\right), \mathbf{g}\right)$, and $\left.\mathbf{h}\right)$ the green circle shows aperture of $2^{\prime \prime}$ radius, while the red circle marks the aperture used by the PHAT team for photometry.

Papers I-IV), the derivation of the physical parameters (age, mass, extinction, $E(B-V)$, and metallicity, $[\mathrm{M} / \mathrm{H}])$ of a given observed star cluster is based on a comparison of its integrated broad-band photometry to a four-dimensional (4D) grid (for the age, mass, extinction, and metallicity) of star cluster models. Each node of the grid contains 1000 star cluster models. Each star cluster model is built by randomly sampling the stellar mass according to the IMF (Kroupa 2001), following the method described in Deveikis et al. (2008; see also Santos \& Frogel 1997; Cerviño et al. 2002). The luminosities of clusters were derived using the PADOVA isochrones from Marigo et al. (2008) with the addition of the thermally pulsing asymptotic giant branch (TP-AGB) phase from Girardi et al. (2010).

The grid was built according to the following nodes: from $\log _{10}(t / \mathrm{yr})=6.6$ to 10.1 in steps of 0.05 , from $\log _{10}\left(M / M_{\odot}\right)=2$ to 7 in steps of 0.05 , and for 13 metallicities: from $[\mathrm{M} / \mathrm{H}]=-2.2$ to +0.2 in steps of 0.2 . This resulted in a grid of 71 values of age and 101 values of mass, with 1000 models per node, hence $\sim 7 \times 10^{6}$ models for each metallicity. To limit the number of models that need to be stored in computer memory, the extinction was computed every time the observed cluster was compared with the grid of models. This ranges from $E(B-V)=0$ to 1 in steps of 0.01 , therefore 101 values for the extinction. We used the standard Cardelli et al. (1989) extinction law derived for the Milky Way, with $R_{V}=3.1$.

We evaluated the likelihood of each node of the grid to represent the magnitudes of a given observed cluster. Within each node, we first computed the likelihood of each of the 1000 star cluster models by

$L_{\text {model }}=\prod_{f=1}^{F} \frac{1}{\sqrt{2 \pi} \sigma_{f}} \exp \left[-\frac{\left(\operatorname{mag}_{f, \text { obs }}-\operatorname{mag}_{f, \text { model }}\right)^{2}}{2 \sigma_{f}^{2}}\right]$,

1 https://archive.stsci.edu/prepds/phat/datalist.html where $f$ stands for one particular filter, $\sigma_{f}$ - for the observation error in the filter $f, \operatorname{mag}_{f}$ - for the observed and model magnitudes in that filter, and $F$ - for the total number of filters. For the WFC3+ACS photometric system we use in this study $F=6$. Then the likelihood of the node of age $t$, mass $M$, extinction $E(B-V)$, and metallicity $[\mathrm{M} / \mathrm{H}]$ is the sum of the likelihoods of its models,

$L_{\text {node }}(t, M, E(B-V),[\mathrm{M} / \mathrm{H}])=\sum_{n=1}^{N} L_{\text {model }, n}$,

where $N=1000$, the total number of models contained in the node. The procedure is repeated for each node of the 4D grid, and the observed star cluster is then classified with the parameters of the node, which maximizes the quantity $L_{\text {node }}$.

More details of this method are given and results of the artificial star cluster classification are discussed in Paper III.

\section{Application to the M31 PHAT star clusters}

\subsection{Selection of the cluster sample}

In this paper we analyse the final catalogue of 2753 clusters of Johnson et al. (2015). In order to select the most reliable clusters and to reject those with poor photometry or artifacts in data, we needed to analyse clusters visually. For this we created individual charts for all clusters, as those shown in Figs. 1-3 for the cluster ID27.

We visually analysed ACS+WFC3 frames and for further consideration accepted only star clusters possessing a minimum of three image defect-free main passbands (WFC3/F336W, $\mathrm{ACS} / F 475 W$, and $\mathrm{ACS} / F 814 W$ ). In the cases of clusters with only these three passbands available, they are accepted if visual classification allows for identification of their type (for example, young, old, massive, etc.). The clusters strongly affected 
(Z free) $: 7.5,3,7,0.2,-0.2$
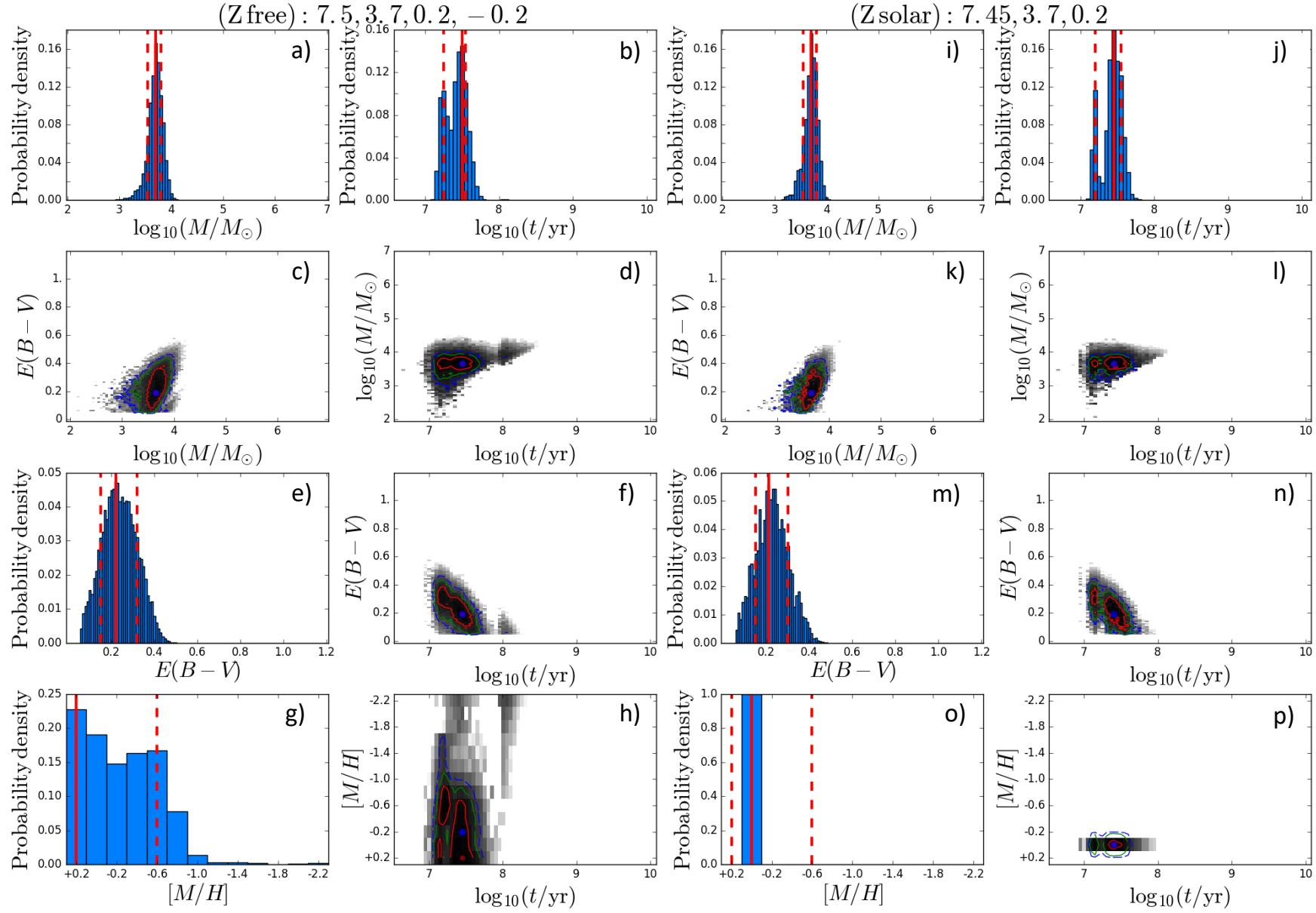

Fig. 2. Diagrams with classification results of the cluster ID27 in free (panels a)-h)) and in fixed solar metallicity (panels i)-p)) cases. The maximum likelihood in 4D parameter space is indicated in the 2D likelihood maps (panels c), d), f), h), k), l), n), p)) by a blue dot, and is surrounded by contours enclosing the $99 \%$ (blue line), 95\% (green line), and 68\% (red line) likelihood levels.

by the presence of bright stars - likely from the foreground or background (usually red stars) - within photometry aperture are rejected.

For the analysis we selected two samples of star clusters. The first one, with a high photometric accuracy, contains 181 objects (group 1). In this sample, the UV passbands (WFC3/F275W and WFC $3 / F 336 W$ ) have uncertainties below $0.15 \mathrm{mag}$, the optical passbands (ACS/F475W and ACS/F814W) - below $0.1 \mathrm{mag}$, and the near-infrared (WFC3/F110W and WFC3/F $160 W$ ) - below $0.2 \mathrm{mag}$, as indicated in Fig. 4.

The second sample of clusters with lower photometric accuracy contains clusters with a maximum uncertainty of 0.4 mag (group 2). In the catalogue of Johnson et al. (2015) the uncertainties of some passbands are unavailable, as they are estimated to be higher than 0.4 mag by the authors. We simply rejected the passbands with unavailable photometric uncertainties or affected by various image defects. Finally, the second sample of clusters contains 1182 objects (group 2), and both groups - 1363 star clusters in total.

\subsection{Derivation of the cluster parameters}

As in Paper III, we analysed the selected cluster sample by firstly fixing the metallicity of all clusters to the solar value, and a second time by allowing a large range of metallicities in the model grid with 13 values of $[\mathrm{M} / \mathrm{H}]=-2.2$ to +0.2 , in steps of 0.2. For clusters with insufficient photometric accuracy, the metallicity was fixed to solar, as it was shown to be a reasonable choice for most of the clusters in Fouesneau et al. (2014) and Paper III.

In Fig. 5 we display the metallicity derived for clusters of groups 1 and 2 for which all six WFC3+ACS passbands are available. Young clusters, below $1 \mathrm{Gyr}$, an overwhelming amount of group 1 and group 2 clusters have supersolar/solar metallicity. As discussed in Paper III, this is partially encouraged by a limit of range effect in metallicity. The overdensity ( $>8 \%$ of the group- 2 objects) at the metal-poor end in panel (a) is also a sign of such an effect, which is due to a lack of photometric accuracy. This feature is less prominent $(<3 \%)$ for the group- 1 clusters, which have better photometric accuracy (see also Paper II, for a study of that effect in different photometric systems). Clusters older than $1 \mathrm{Gyr}$ are more distributed, with an over-density around $[\mathrm{M} / \mathrm{H}]=-0.5$ (Fig. $5 \mathrm{~d}$ ).

The metallicity distributions derived for the subsamples of 91 (group 1) and 261 (group 2) clusters younger than $1 \mathrm{Gyr}$ (see Figs. 5a and c) provide support for the idea of fixing the metallicity for such clusters to solar, and derive the metallicity only for older ones.

In the following analysis we adopt this choice for the parameter derivation; for clusters younger than $1 \mathrm{Gyr}$ the metallicity is fixed to solar, while for older clusters the metallicity is free. The derived parameters are shown in Fig. 6 for the age, mass, 

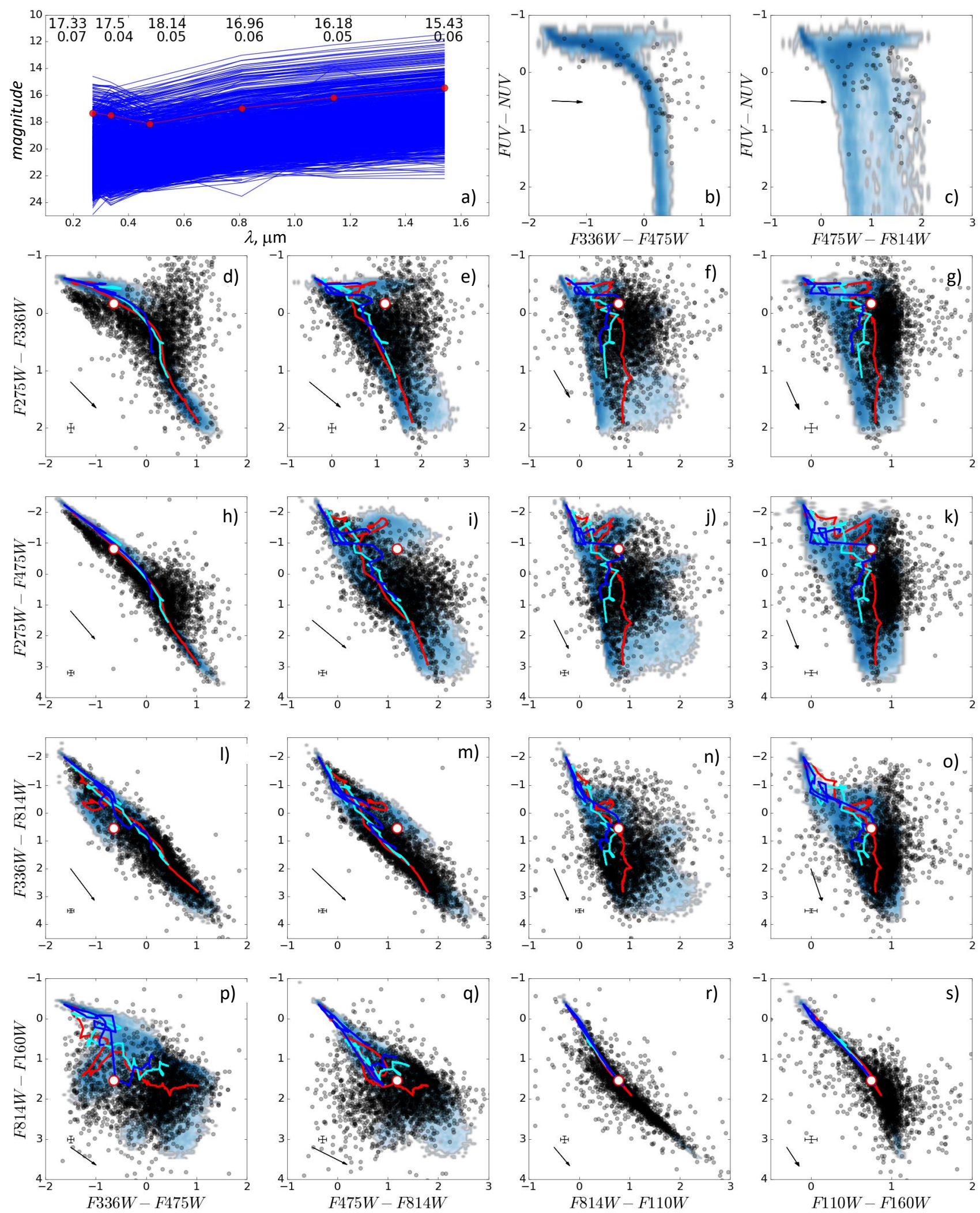

Fig. 3. Photometric diagrams of the cluster ID27 (large white dot). Panel a) shows the WFC+ACS passband magnitudes (in red) of the cluster ID27 compared to all other clusters from the catalogue (in blue) vs. wavelength. Magnitudes and their uncertainties are indicated in the upper part of the panel. Panels b) and c) show the GALEX FUV-NUV colour vs. WFC3+ACS colours for clusters with available GALEX photometry from Kang et al. (2012). Panels (d)-s)) show colour-colour diagrams in WFC3+ACS passbands using the PHAT photometry. On $y$-axes (panels d), h), l), p)) and on $x$-axes (panels p), q), r), s)) colour combinations go from blue to red. In each colour-colour diagram 3 SSP (single stellar population) lines for solar $([\mathrm{M} / \mathrm{H}]=0$, red), intermediate $([\mathrm{M} / \mathrm{H}]=-1$, cyan $)$, and poor $([\mathrm{M} / \mathrm{H}]=-2$, blue) metallicities are shown. Arrows indicate extinction vectors corresponding to $A_{V}=1$. Error bars indicate observation errors of colours. 

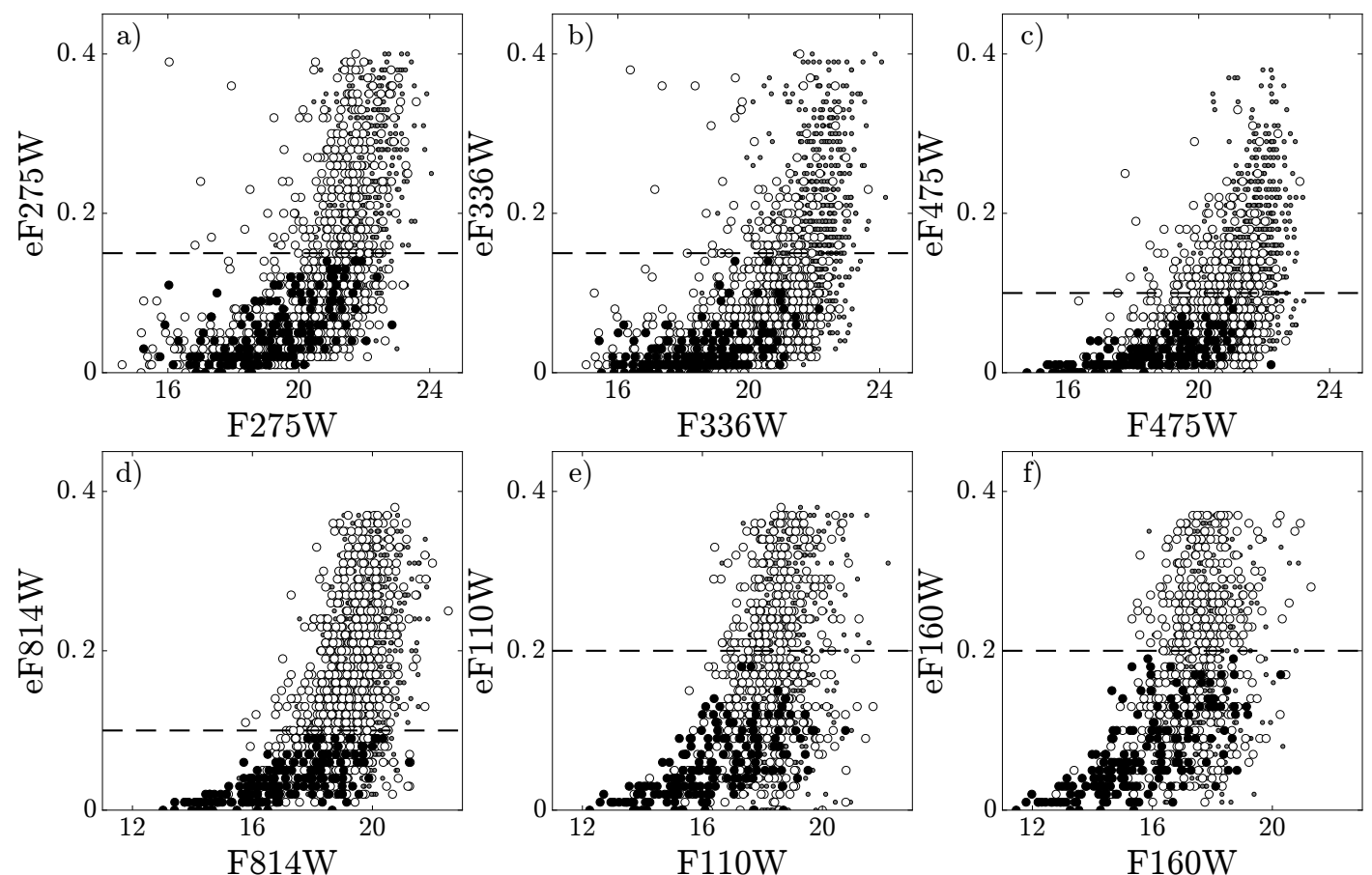

Fig. 4. Photometric accuracy of the studied sample of star clusters from the Johnson et al. (2015) catalogue. The small grey dots mark the clusters rejected during the visual classification (see text) and not studied in the following analysis. Group 1 clusters (181 objects, large filled dots) have upper limits of the photometric errors indicated by long dashed lines in different filters. Group 2 clusters (1182 objects, large open dots) have photometric errors larger than these limits in at least one filter.

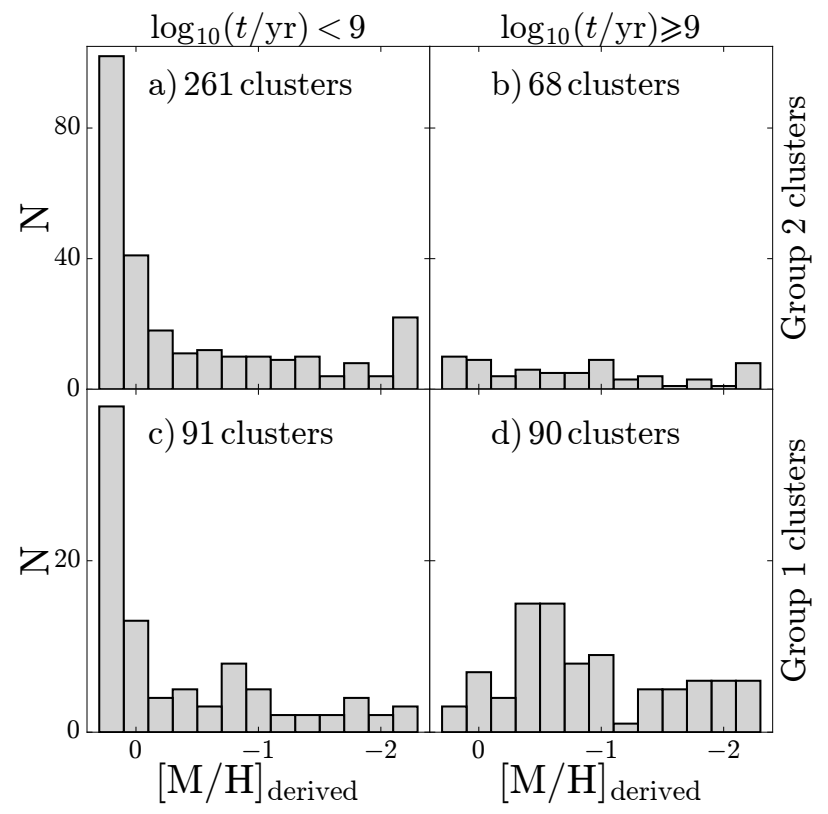

Fig. 5. Derived metallicity of clusters with ages lower than $1 \mathrm{Gyr}$ (panels a), c)) and of clusters with ages higher than 1 Gyr (panels b), d)), for group 2 (top panels) and group 1 (bottom panels) clusters.

and extinction for the whole cluster sample (1363 star clusters) studied in this paper (Table 1).

Table 1, available at the CDS, contains the following information. Col. 1 lists the PHAT name of the cluster, Cols. 2 and 3 give coordinates RA (2000) and Dec (2000), Col. 4 gives PHAT magnitude in the passband $F 475 W$, Cols. 5-7 give cluster parameters derived by assuming solar metallicity $\left(\log _{10}(t / \mathrm{yr})\right.$, $\log _{10}\left(M / M_{\odot}\right)$, and $\left.E(B-V)\right)$, Cols. 8-11 give cluster parameters
Table 2. Colour excess ratios used to calculate $Q$ parameters.

\begin{tabular}{cc}
\hline \hline Colour excess ratio & Value \\
\hline$E(F 275 W-F 336 W) / E(F 475 W-F 814 W)$ & 0.64 \\
$E(F 475 W-F 814 W) / E(F 110 W-F 160 W)$ & 4.85 \\
\hline
\end{tabular}

derived together with metallicity $\left(\log _{10}(t / \mathrm{yr}), \log _{10}\left(M / M_{\odot}\right)\right.$, $E(B-V)$, and $[\mathrm{M} / \mathrm{H}])$.

\subsection{Sanity check using reddening-free $Q$ parameters}

The reddening-free $Q$ parameter is a combination of two colour indices (involving three or four passbands) with the ratio of the two associated colour excesses, meaning that its value should not depend on the reddening, provided that the extinction law is well known (see, e.g. Massey 1998; Bianchi \& Efremova 2006). A general expression, using four passbands $m_{1}, m_{2}, m_{3}$, and $m_{4}$ is

$Q_{m_{1} m_{2} m_{3} m_{4}}=\left(m_{1}-m_{2}\right)-\frac{E\left(m_{1}-m_{2}\right)}{E\left(m_{3}-m_{4}\right)}\left(m_{3}-m_{4}\right)$.

The classical $Q$ parameter, constructed with passbands $U, B$, and $V$, has been extensively used in the last decades for the study of stellar populations. In this study, we propose the use of $Q$ parameters of star clusters as a suitable way to check some of their derived parameters. Colour excess ratios used to calculate $Q$ parameters are given in Table 2 (Cardelli et al. 1989).

Figure 7 shows a $Q$ parameter and a difference of $Q$ parameters vs. the metallicity for both SSP models and cluster data with ages beyond 1 Gyr. In both panels the majority of globularlike clusters (red dots) follow the same trend as the SSP models. For other clusters the correlation is less strong, likely due to the lower signal-to-noise ratio (lower photometric accuracy) and hence less secure metallicity derivation. We note that for a fixed 

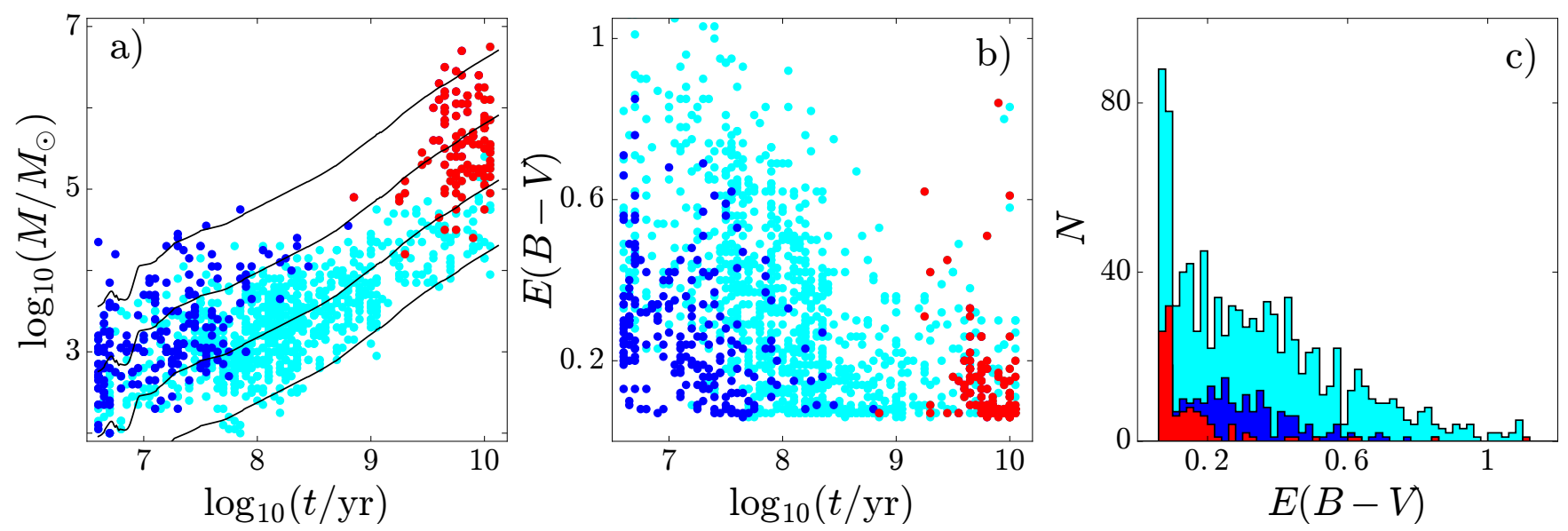

Fig. 6. Parameters derived for 1363 star clusters: mass vs. age (panel a)), extinction vs. age (panel b)), and extinction histogram (panel c)). The clusters are colour-coded according to their type: bright in UV $(F 275 W<19$, blue), faint in UV ( $F 275 W \geq 19$, cyan), globular clusters (red). In panel a) four SSP lines are shown for fixed absolute magnitudes $F 475 W=-3,-5,-7$, and -9 from bottom to top.
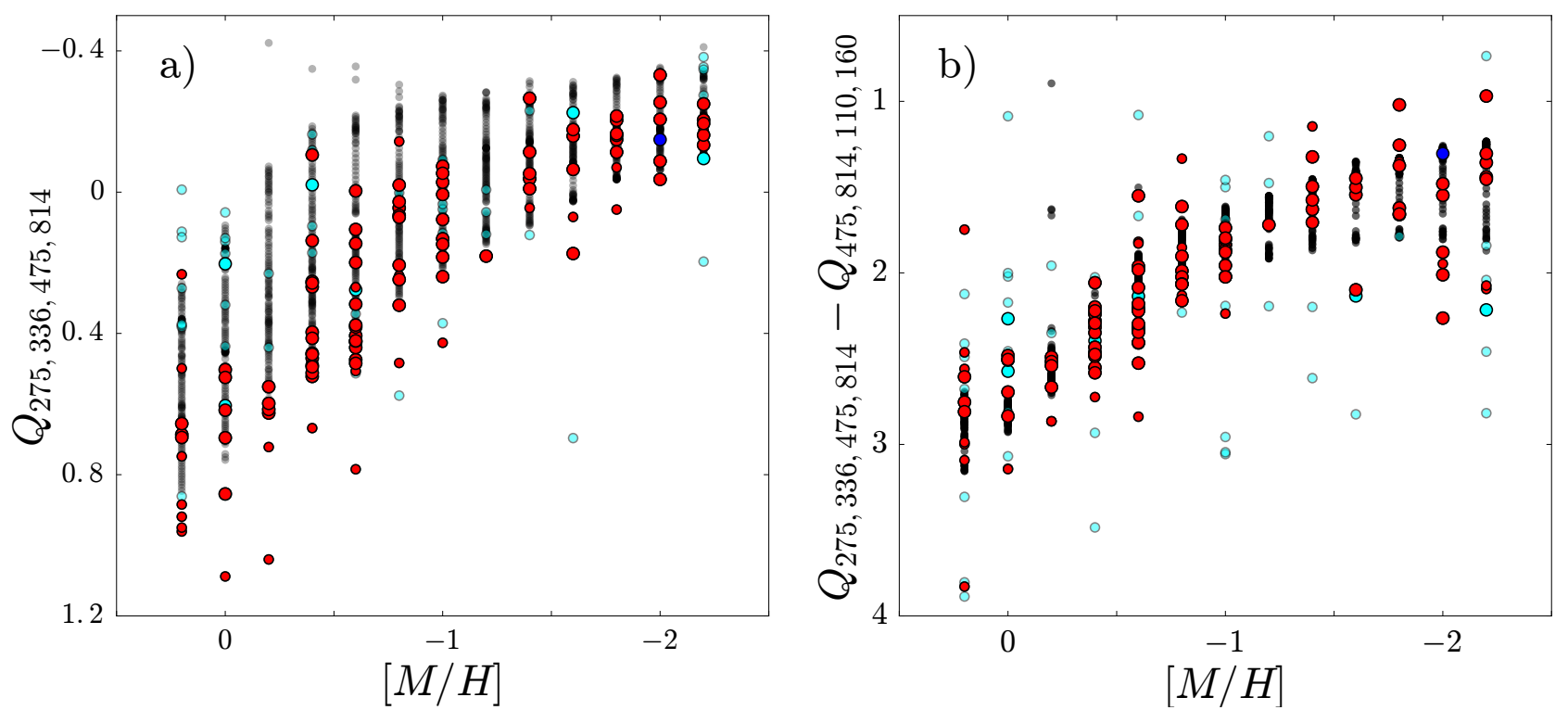

Fig. 7. $Q$ parameter (panel a)) and $Q-Q$ parameters (panel b)) vs. metallicity diagrams for clusters with age derived beyond $1 \mathrm{Gyr}$. The clusters are colour-coded according to their type, as in Fig. 6. Large dots are for group 1 while small dots are for group 2 clusters. Black dots are SSP models with age extending from $1 \mathrm{Gyr}$ to $13 \mathrm{Gyr}$, downwards in panel a) and upwards in panel b)).

metallicity, the age of SSP models goes from $1 \mathrm{Gyr}$ to $13 \mathrm{Gyr}$ downwards in panel (a), while upwards in panel (b). The case of difference of $Q$ parameters (panel b) is especially interesting because it allows us to disentangle SSP models of different metallicities. Conversely, when using only a single $Q$ parameter (panel a) the effect of age slightly prevents such an unambiguous metallicity determination.

\subsection{Comparison with spectroscopic studies}

Using spectroscopy Caldwell et al. (2009, 2011) derived the age, mass, and extinction of young clusters $(<1 \mathrm{Gyr})$ and Caldwell et al. (2011) derived the mass, extinction, and metallicity of old clusters (fixing the age of most of them to $14 \mathrm{Gyr}$; the individual age was derived only for a few clusters). Here we use updated catalogues as of April 2016.

The age and mass derived in this work are found in reasonable agreement with the parameters derived for common clusters by Caldwell et al. (2009, 2011) for both young and old clusters, as shown in Fig. 8. However, the extinction derived in our study only loosely correlates with the reddening values estimated by Caldwell et al. (2009, 2011) again for both young and old clusters.

For clusters with high enough photometric accuracy we derived the metallicities, which are in agreement with the spectroscopically derived parameters by Caldwell et al. (2011), as shown in Fig. 9 for both young and old clusters. Therefore, it remains puzzling, why the age, mass, and metallicity of clusters derived consistently from photometric and spectroscopic observations are in reasonable agreement, while extinction is not.

\section{Properties of the star cluster sample}

Figure 10 shows the derived age and mass distributions (panels a, b) as well as the age and mass functions (panels c, d). The age function (panel c) is composed of a two-slope profile $-\mathrm{a}$ feature also observed in the cluster populations of other galaxies. Boutloukos \& Lamers (2003) and Lamers et al. (2005) 

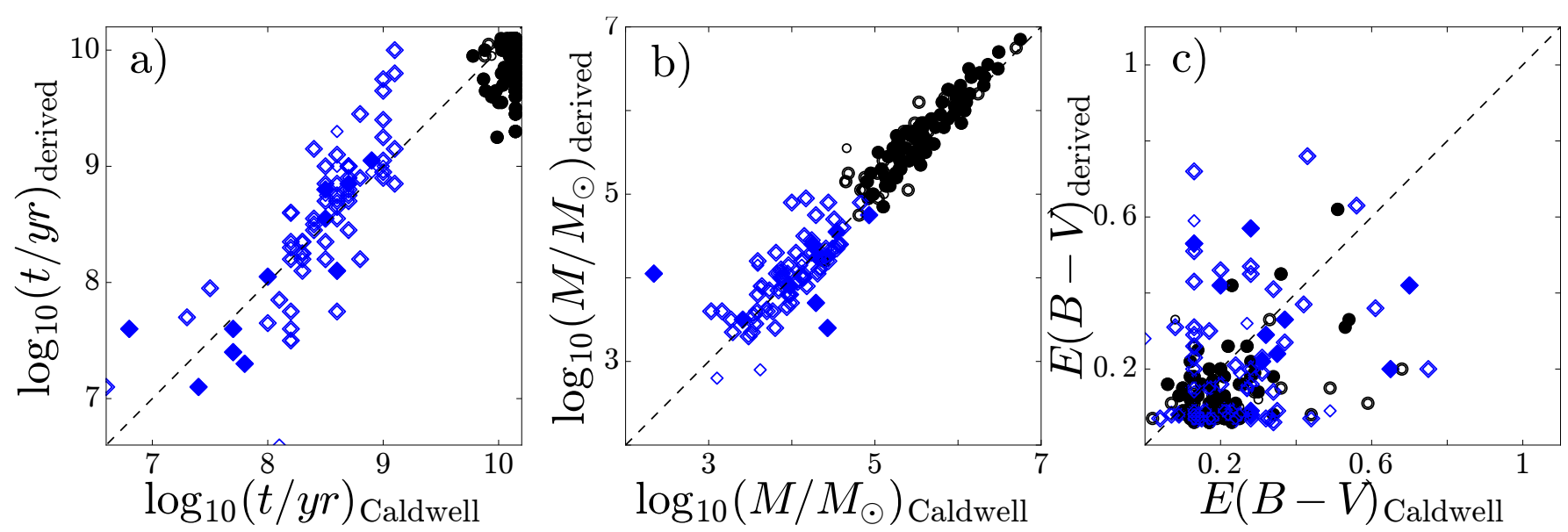

Fig. 8. Cluster parameters derived in this study vs. those of Caldwell et al. (2009) for young (open and filled blue diamond symbols) clusters and vs. those of Caldwell et al. (2011) for old (open and filled black dots) clusters, shown for the age (panel a)), the mass (panel b)), and the extinction (panel c)). Filled symbols mark the group 1 clusters and open symbols mark the group 2 clusters, as defined in Fig. 4.

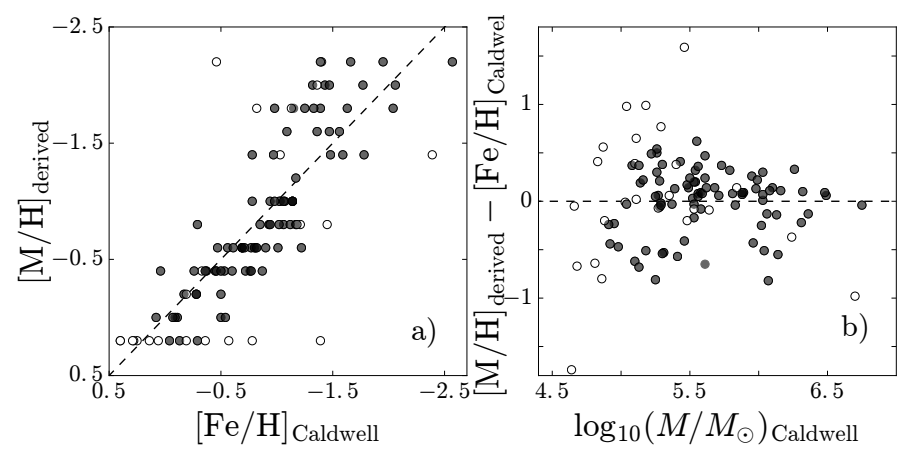

Fig. 9. Metallicity derived in this study vs. Caldwell et al. (2011) for old clusters (panel a)). Panel b) shows the difference of metallicity values derived in this study and in Caldwell et al. (2011) vs. their derived cluster mass. Filled dots mark the group 1 clusters and open dots mark the group 2 clusters, as defined in Fig. 4.

interpreted the first slope as a natural magnitude fading as a result of stellar evolution, and the second slope as being due to cluster disruption by the galaxy tidal field or encounters with giant molecular clouds. Hence we see here that the cluster sample is dominated by the magnitude fading until $\log _{10}(t / \mathrm{yr}) \sim 8.3$ and that later the cluster disruption phase takes over. A slightly later transition age of $\log _{10}(t / y r) \sim 8.5$ was derived for the star-cluster population in the southwest field of the M31 galaxy by Vansevičius et al. (2009), using star cluster photometry data from Narbutis et al. (2008), and for the star cluster population of the Local Group M 33 galaxy (de Meulenaer et al. 2015b). Following Gieles (2009), we compare the cluster differential mass distribution to the Schechter (1976) function, defined as

$\mathrm{d} N / \mathrm{d} M=A \times M^{-\beta} \times \exp \left(-M / M^{*}\right)$,

where the $A$ constant scales with the cluster formation rate, $\beta$ is the power-law index of the mass function, and $M^{*}$ stands for the characteristic mass.

As was found for spiral galaxies (see, e.g. Larsen 2009; Portegies Zwart et al. 2010), the derived mass function of the star cluster sample follows Eq. (4) with $\beta=2$ and $M^{*}=$ $2 \times 10^{5} M_{\odot}$. We adapted these values of the parameters and arbitrarily assumed a scaling constant $A$ in order to compare visually with the cluster differential mass distribution, see Fig. 10d.

In a recent review, Adamo et al. (2015) stressed that in most observational studies the age function of clusters shows an
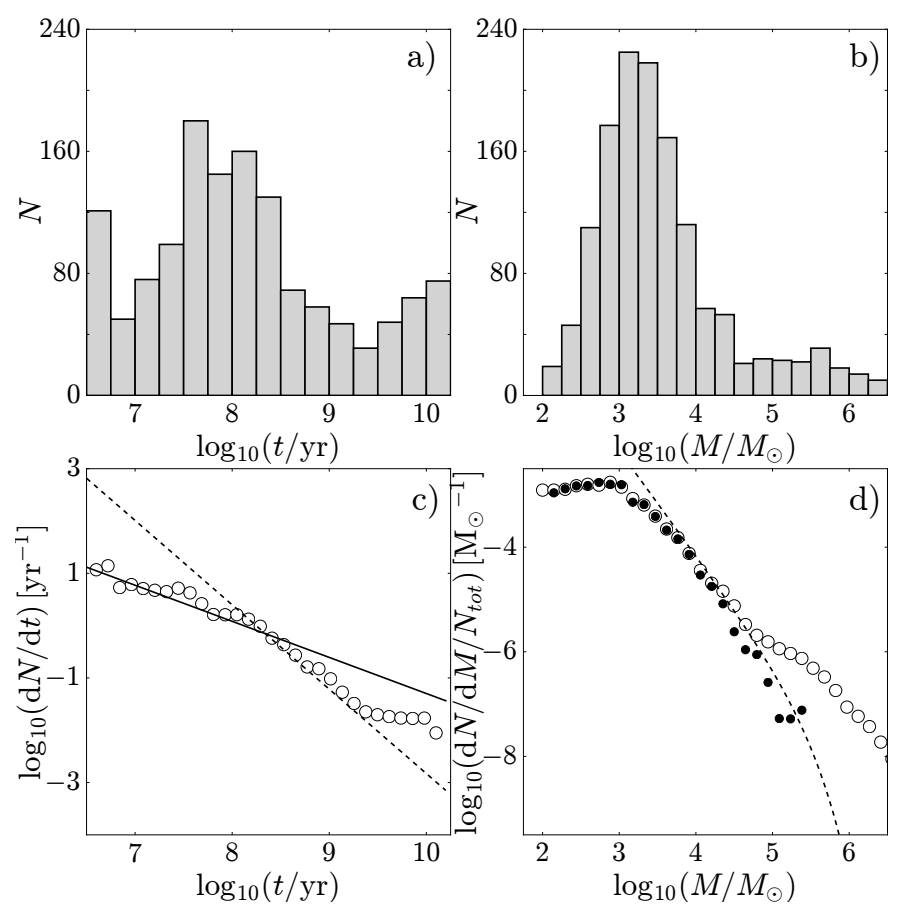

Fig. 10. Properties of the star-cluster sample. Top panels show age (panel a)) and mass (panel b)) distributions of the studied clusters. Bottom panels show age (panel c)) and mass (panel d)) differential distributions. In panel c) the mass is truncated to $\log _{10}\left(M / M_{\odot}\right)=3$. In panel d) open dots represent mass function for all clusters, while filled dots represent mass function for clusters younger than $3 \mathrm{Gyr}$, to exclude massive globular-like clusters.

almost constant age distribution before $\sim 100 \mathrm{Myr}$ and a drastic decrease above this age due to the combination of cluster disruption as well as sample incompleteness. They noted also that this agrees with theoretical expectations, which rule out rapid cluster disruption for ages younger than $100 \mathrm{Myr}$ in most galaxies.

In Fig. 11 we show that the mass function of the studied sample is compatible with the mass function of other galaxies on a wide cluster mass range, following the Schechter (1976) function with a characteristic mass of approximately $10^{5} M_{\odot}$. However, one should keep in mind that selection effects are not taken 


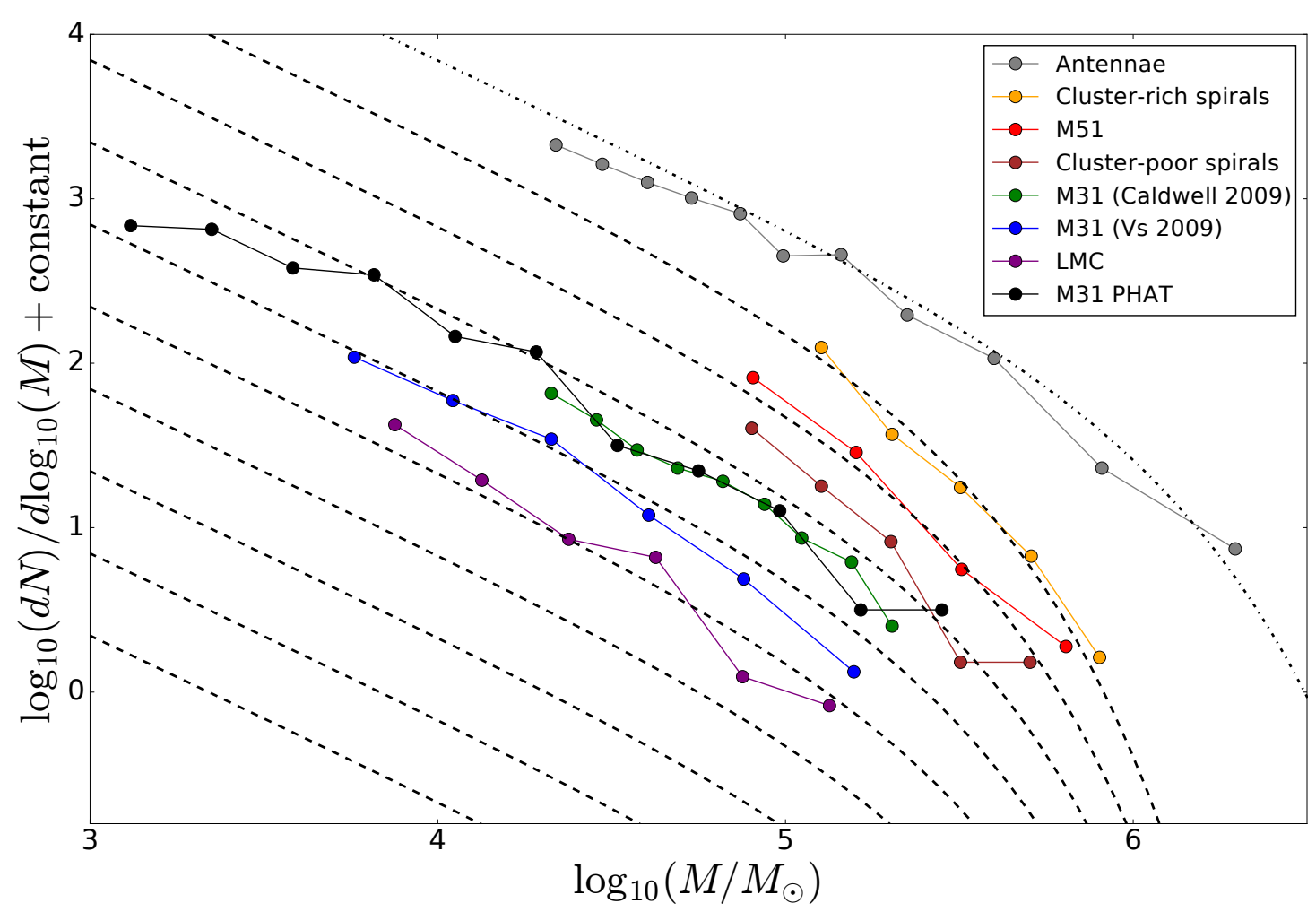

Fig. 11. Star cluster mass functions of different galaxies (Portegies Zwart et al. 2010) and the cluster mass function of M 31 derived in this paper. Data from Vansevičius et al. (2009) are marked in the legend as "Vs 2009".

into account, and can produce strong biases, especially at the low mass limit of the sample.

\section{Conclusions}

In this paper we:

- studied 2753 star clusters from the M31 PHAT cluster catalogue (Johnson et al. 2015);

- derived the parameters for 1363 star clusters and found consistent agreement with spectroscopic studies of the same clusters by Caldwell et al. (2009, 2011), Caldwell \& Romanowsky (2016) for both young and old objects;

- showed that the disruption of star clusters in the North-East region of M 31 is negligible before $\sim 300 \mathrm{Myr}$, in agreement with Vansevičius et al. (2009), who studied star clusters in the South-West region of M 31;

- showed that the mass function of studied star clusters is compatible with mass functions derived in other spiral galaxies on a wide cluster-mass range and follows the Schechter mass function with a characteristic mass around $10^{5} M_{\odot}$.

Acknowledgements. We are grateful to the anonymous referee who helped improve the paper. We acknowledge the intense use of the Topcat software and ipython notebooks. This research was funded by a grant (No. LAT-09/2016) from the Research Council of Lithuania.

\section{References}

Adamo, A., Kruijssen, J. M. D., Bastian, N., Silva-Villa, E., \& Ryon, J. 2015, MNRAS, 452, 246

Beerman, L. C., Johnson, L. C., Fouesneau, M., et al. 2012, ApJ, 760, 104 Bianchi, L., \& Efremova, B. V. 2006, AJ, 132, 378
Boutloukos, S. G., \& Lamers, H. J. G. L. M. 2003, MNRAS, 338, 717

Caldwell, N., \& Romanowsky, A. J. 2016, ApJ, 824, 42

Caldwell, N., Harding, P., Morrison, H., et al. 2009, AJ, 137, 94

Caldwell, N., Schiavon, R., Morrison, H., Rose, J. A., \& Harding, P. 2011, AJ, 141,61

Cardelli, J. A., Clayton, G. C., \& Mathis, J. S. 1989, ApJ, 345, 245

Cerviño, M., Valls-Gabaud, D., Luridiana, V., \& Mas-Hesse, J. M. 2002, A\&A, 381,51

Dalcanton, J. J., Williams, B. F., Lang, D., et al. 2012, ApJS, 200, 18

de Meulenaer, P., Narbutis, D., Mineikis, T., \& Vansevičius, V. 2013, A\&A, 550, A20 (Paper I)

de Meulenaer, P., Narbutis, D., Mineikis, T., \& Vansevičius, V. 2014, A\&A, 569, A4 (Paper II)

de Meulenaer, P., Narbutis, D., Mineikis, T., \& Vansevičius, V. 2015a, A\&A, 574, A66 (Paper III)

de Meulenaer, P., Narbutis, D., Mineikis, T., \& Vansevičius, V. 2015b, A\&A, 581, A111 (Paper IV)

Deveikis, V., Narbutis, D., Stonkutè, R., Bridžius, A., \& Vansevičius, V. 2008, Baltic Astronomy, 17, 351

Fouesneau, M., \& Lançon, A. 2010, A\&A, 521, A22

Fouesneau, M., Johnson, L. C., Weisz, D. R., et al. 2014, ApJ, 786, 117

Gieles, M. 2009, MNRAS, 394, 2113

Girardi, L., Williams, B. F., Gilbert, K. M., et al. 2010, ApJ, 724, 1030

Johnson, L. C., Seth, A. C., Dalcanton, J. J., et al. 2012, ApJ, 752, 95

Johnson, L. C., Seth, A. C., Dalcanton, J. J., et al. 2015, ApJ, 802, 127

Kang, Y., Rey, S.-C., Bianchi, L., et al. 2012, ApJS, 199, 37

Kroupa, P. 2001, MNRAS, 322, 231

Lamers, H. J. G. L. M., Gieles, M., \& Portegies Zwart, S. F. 2005, A\&A, 429, 173

Larsen, S. S. 2009, A\&A, 494, 539

Marigo, P., Girardi, L., Bressan, A., et al. 2008, A\&A, 482, 883

Massey, P. 1998, ApJ, 501, 153

Narbutis, D., Vansevičius, V., Kodaira, K., Bridžius, A., \& Stonkutė, R. 2008, ApJS, 177, 174

Portegies Zwart, S. F., McMillan, S. L. W., \& Gieles, M. 2010, ARA\&A, 48, 431

Santos, Jr., J. F. C., \& Frogel, J. A. 1997, ApJ, 479, 764

Schechter, P. 1976, ApJ, 203, 297

Vansevičius, V., Kodaira, K., Narbutis, D., et al. 2009, ApJ, 703, 1872

Weisz, D. R., Fouesneau, M., Hogg, D. W., et al. 2013, ApJ, 762, 123

Zurita, A., \& Bresolin, F. 2012, MNRAS, 427, 1463 